\title{
A Theoretical Analysis of Camera Response Functions in Image Deblurring
}

\author{
Xiaogang Chen ${ }^{1,2,3}$, Feng Li ${ }^{3}$, Jie Yang ${ }^{1,2}$, and Jingyi $\mathrm{Yu}^{3}$ \\ 1 Shanghai Jiao Tong University, Shanghai, China. \\ 2 Key Laboratory of System Control and Information Processing, \\ Ministry of Education, Shanghai, China. \{cxg, jieyang $\}$ @sjtu.edu.cn \\ 3 University of Delaware, Newark, DE, USA. \{feli,yu\}@cis.udel.edu
}

\begin{abstract}
Motion deblurring is a long standing problem in computer vision and image processing. In most previous approaches, the blurred image is modeled as the convolution of a latent intensity image with a blur kernel. However, for images captured by a real camera, the blur convolution should be applied to scene irradiance instead of image intensity and the blurred results need to be mapped back to image intensity via the camera's response function $(\mathrm{CRF})$. In this paper, we present a comprehensive study to analyze the effects of CRFs on motion deblurring. We prove that the intensity-based model closely approximates the irradiance model at low frequency regions. However, at high frequency regions such as edges, the intensity-based approximation introduces large errors and directly applying deconvolution on the intensity image will produce strong ringing artifacts even if the blur kernel is invertible. Based on the approximation error analysis, we further develop a dualimage based solution that captures a pair of sharp/blurred images for both CRF estimation and motion deblurring. Experiments on synthetic and real images validate our theories and demonstrate the robustness and accuracy of our approach.
\end{abstract}

\section{Introduction}

Image deblurring is a long standing problem in computer vision and image processing. A common assumption in most existing approaches is that a blurred image is the convolution result of a blur-free intensity image $I$ with a blur kernel $K$, i.e., $\widehat{B}=I \otimes K$. However, for images captured by a real camera, the blur convolution should be applied to image irradiance $\widetilde{I}$ rather than image intensity. The blurred results then need to be mapped back to image intensity via the camera's response function $(\mathrm{CRF}) \psi$ as:

$$
B=\psi(\widetilde{I} \otimes K) .
$$

Eqn. (1) reveals that, unless the CRF $\psi$ is linear, the actual captured blur image $B$ will be different from the synthetically blurred intensity image $\widehat{B}$. The correct way to deblur $B$ hence is to first map $B$ back to irradiance as $\psi^{-1}(B)$, then 
apply deconvolution, and finally map it back to intensity. In reality, recovering the CRF $\psi$ of a real camera often requires applying complex calibration processes [1] or using special scene settings [2].

Previously irradiance domain deconvolution methods assume a known CRF curve $[3,4]$. More recently approaches [5-8] apply additional constraints in the intensity domain to reduce visual artifacts. Although they simplify the estimation, they may introduce significant approximation errors caused by the underlying linear CRF assumption. More important, it is desirable to characterize how close the intensity based convolution is to the ground truth irradiance blurring model and where the errors are large.

In this paper, we present a comprehensive study to analyze the effect of CRFs on motion deblurring. Our contributions are two-folded. On the theory side, we prove that the intensity-based blur model closely approximates the irradiancebased one at low frequency regions. We further derive a closed-form error bound to quantitatively measure the difference between $B$ and $\widehat{B}$. However, at high frequency regions such as near texture or occlusion edges, the intensity-based approximation introduces large errors and directly applying deconvolution on the intensity image introduces ringing artifacts even if the blur kernel is invertible.

On the application front, we develop a simple but effective computational photography technique to recover the CRF. Our approach is inspired by the recent single-image based CRF estimation method [9] that strategically blurs the image under 1D linear motion. We, instead, capture a pair of sharp/blurred images and directly use motion blurs caused by hand shakes. Specifically, we first automatically align the two images and then use the sparsity prior to recovering the blur kernel. To recover the CRF, we represent it using the Generalized Gamma Curve Model (GGCM) and find the optimal one by fitting pixels near edge regions in the two images. Experiments on synthetic and real images validate our theories and demonstrate that our dual-image approach is robust and accurate.

Concurrent with this research, Kim et al. [10] independently developed a similar analysis to characterize nonlinear CRFs in motion blur and proposed a single-image deblurring scheme. We, on the other side, focus on the theoretical analysis of the approximation errors and derive an error bound.

\section{Related Work}

Our work is related to a number of areas in image processing and computational imaging.

CRF Estimation. The CRF can be estimated by capturing multiple images from the same viewpoint with different but known exposure settings. The pioneer work by Debevec and Malik [1] assume smooth CRFs and form an overdetermined linear system for simultaneously estimating the irradiance at each pixel as well as the inverse CRF. Later approaches [11-14] adopt more complex CRF models based on polynomial fitting [13] or Empirical Model of Response (EMoR) [12]. EMoR, for example, is particularly useful for its low dimensionality and high accuracy and has been widely used in various imaging applications 
[11,15-18]. Lee et al. [14] employed this low-rank structure of irradiance whereas Kim and Pollefeys [11] developed a full radiometric calibration algorithm to simultaneously estimate CRF, exposure, and vignetting. More recently [15], they used an outdoor image sequence with varying illumination based CRF estimating method, while different from previous constant illumination methods $[1,12]$.

A downside of multi-image approaches is that both the scene and camera have to be static during capturing. To resolve this issue, a number of singleimage based approaches have been recently proposed. The classical approach uses the Macbeth color chart [2] and assumes known surface reflectance for calibrating the radiometric function. In Lin and Zhang [17] and Lin et al. [16], the authors analyzed the linear blending properties of edges in gray scale or color images. Matsushita and Lin [18] explored how symmetric distribution of noise gets skewed by the CRF and used a single noisy image for recovering the CRF. Wilburn et al. [9] further analyzed how the CRF affects linearly motion blurred edges and then used the blurred edges for sampling the CRF. Their approach requires strategically introducing a linear motion blur whereas we use general motion blurs, e.g., the ones caused by hand shakes.

Image Deblurring. Our work aims to actively use image blurs for recovering the CRF, and also analyzes ringing effect in deblurred images caused by the nonlinearity introduced by the CRF. The literature on image deblurring is huge and we refer the readers to the recent papers $[19,20]$ for a thorough review. Most existing approaches assume that the CRF is known or linear and directly apply the deblurring on the intensity domain. More recent approaches have focused on imposing priors to the kernel or to image statistics to improve quality. For example, Fergus et al. [3] assumed the gradient distribution of a sharp image is heavy-tailed and apply inference to recover the optimally deblurred result. Shan et al. [5] concatenated two piece-wise continuous functions to fit the heavy-tailed distribution and use local image constraint and high-order noise distribution to suppress the ringing artifacts. Other types of priors such as edge sharpness $[21$, 6], transparency [22], kernel sparsity [23] and Fields of Experts [24] have shown promising results for image restoration. Levin et al. [19] analyzed and evaluated many recent blind deconvolution algorithms. Due to the complexity of both estimating CRF and blind deconvolution, recent approaches $[7,6,5]$ attempt to model the blur on image intensities by bypassing the irradiance-intensity conversion. Additional constraints such as minimal ringing can be added to the deconvolution process. However, the underlying approximation error has been barely discussed.

\section{Deblurring: Irradiance vs. Intensity}

We first study the role of the CRF in image blurring/deblurring. Before proceeding, we clarify our notation. $I$ represents the blur-free intensity image, $E$ represents its corresponding scene irradiance, $\Delta t_{I}$ is the exposure time. We also assume that the CRF $\psi$ is monotonically increasing and use $\phi$ to represent its 
inverse. We have $I=\psi\left(E \cdot \Delta t_{I}\right)$ or,

$$
E=\phi(I) / \Delta t_{I}
$$

We use $B$ to represent the ground truth blurred image that is obtained by first convolving the irradiance image $E$ with the blur kernel $K$ and then mapping the result onto intensity as:

$$
B=\psi\left((E \otimes K) \cdot \Delta t_{B}\right),
$$

where all kernel elements in $K$ are non-negative and sum to one. Substituting Eqn.(2) into (3), we have:

$$
B=\psi((\phi(I) \otimes K) \cdot r), r=\Delta t_{B} / \Delta t_{I} .
$$

$r$ represents exposure ratio. It is important to note that Eqn. (4) simplifies the exposure ratio $r$ in terms of the exposure time. In practice, we can factor other exposure parameters such as the ISO and the aperture size ( $F$ number) into $r^{4}$.

In our analysis, we assume that the hypothetic latent image $I$ and the captured blurred image $B$ have identical exposure, i.e., $r=1$, and we have:

$$
B=\psi(\phi(I) \otimes K) .
$$

Actually, two images have the same exposure do not necessitate the same exposure settings of a camera. They may differ in exposure time, ISO value or aperture size ${ }^{4}$.

For blind image deconvolution, the goal is to recover the latent sharp image and the kernel. By Eqn. (5), it is straightforward to map $B$ back to the irradiance domain and then perform the deconvolution. However, it is also popular to use the intensity based convolution model $\widehat{B}=I \otimes K$ in conventional deblurring algorithms $[5,7]$. By using additional edge prediction [6] or gradient regularization [7], the intensity based model is also able to produce pleasing deblurring results. We aim to measure the Blur Inconsistency $\Gamma=B-\widehat{B}$, and to understand where the intensity based convolution model introduces approximation error.

\subsection{Blur Inconsistency}

We first study where Blur Inconsistency occurs.

Claim 1. In uniform intensity regions, $\Gamma=0$.

Since pixels within the blur kernel region have uniform intensity, we have $\phi(I \otimes$ $K)=\phi(I)=\phi(I) \otimes K$. Therefore,

$$
B=\psi(\phi(I) \otimes K)=\psi(\phi(I \otimes K))=\widehat{B},
$$

\footnotetext{
${ }^{4}$ As shown in [4] and [13], the exposure ratio can be calculated by $r=\frac{I S O_{B} \Delta t_{B} F_{I}^{2}}{I S O_{I} \Delta t_{I} F_{B}{ }^{2}}$. $I S O_{I}$ and $F_{I}$ denote the camera ISO setting and aperture F-number relating to image $I$. $I S O_{B}$ and $F_{B}$ are the corresponding values with respect to image $B$.
} 
thus $\Gamma=0$.

Claim 1 applies to any (non-linear) CRF. This also implies that uniform regions in $B$ will not be useful to recover the CRF.

Claim 2. If the blur kernel $K$ is small and the $C R F \psi$ is smooth, $\Gamma \approx 0$ in low frequency regions in $I$.

Proof. Let $I=\bar{I}+\Delta I$ in a local patch covered by the blur kernel $K . \bar{I}$ is the average intensity within the patch and $\Delta I$ is the deviation from $\bar{I}$. In low frequency regions, $\Delta I$ is small.

Next we apply the first-order Taylor expansion to $\phi(I) \otimes K$ as:

$$
\phi(\bar{I}+\Delta I) \otimes K \approx \phi(\bar{I}) \otimes K+\left(\phi^{\prime}(\bar{I}) \cdot \Delta I\right) \otimes K
$$

Since $\bar{I}$ is uniform, we have $\phi(\bar{I}) \otimes K=\phi(\bar{I})$, and $\phi^{\prime}(\bar{I})$ is constant in the local neighborhood. The right hand side (RHS) of Eqn.(6) thus can be approximated as:

$$
\phi(\bar{I})+\phi^{\prime}(\bar{I}) \cdot \Delta I \otimes K .
$$

Furthermore, since $\phi(I \otimes K)=\phi(\bar{I} \otimes K+\Delta I \otimes K)$, by using the first-order Taylor expansion, we have

$$
\phi(I \otimes K) \approx \phi(\bar{I} \otimes K)+\phi^{\prime}(\bar{I} \otimes K) \cdot(\Delta I \otimes K) .
$$

Since $\bar{I}$ is constant in the local patch, the RHS of (8) is equal to:

$$
\phi(\bar{I})+\phi^{\prime}(\bar{I}) \cdot \Delta I \otimes K .
$$

Therefore,

$$
B=\psi(\phi(I) \otimes K) \approx \psi(\phi(I \otimes K))=\widehat{B},
$$

i.e., $\Gamma \approx 0$.

Claim 2 holds only for small kernels. If the kernel is large, like $80 \times 80$, our first-order Taylor expansion will be less accurate. To illustrate this property we simulate $I, \widehat{B}$ and $B$ on a $1 \mathrm{D}$ signal with $\phi$ being a gamma 2.2 curve used in [3, 4]. We then plot $\Gamma$ with different sized $K$ s (10, 20, 30 and 80 pixels). Figure 1 (a) shows that $\Gamma \approx 0$ for the first three kernels. Claim 2 also implies that low frequency regions in $I$ contribute little information to the CRF.

Claim 3. $\Gamma$ can be large at high frequency regions in $I$.

Recent analysis $[9,10]$ shows that the blurred edges in $B$ best illustrate the non-linearity of the CRF. When an edge is motion blurred, the results should demonstrate linear changes on intensity due to the linear convolution. However, in the observed image the linear profile is warped by the $\operatorname{CRF}[9,17]$ and causes $B$ to deviate from $I \otimes K$.

Specifically, let us consider a sharp edge in $I$ represented by a Step Edge Function,

$$
I(x)= \begin{cases}0 & x<0.5 \\ 1 & \text { else }\end{cases}
$$




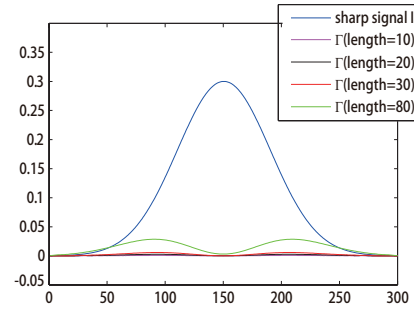

(a)

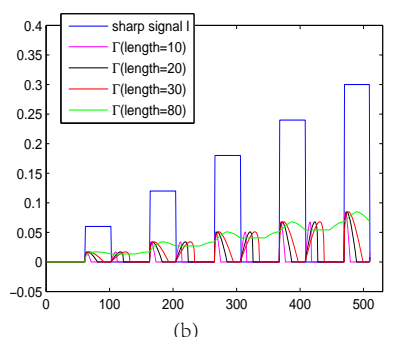

(b)

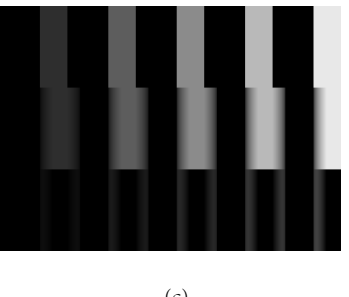

(c)

Fig. 1. Blur Inconsistency $\Gamma$. (a): $\Gamma$ is computed on a 1D sharp signal with a gamma 2.2 CRF curve with four different blur kernels of size 10, 20, 30 and 80 pixels. (b): The diagram of $\Gamma$ vs. different kernel sizes. Blurs are applied to the top row pattern in (c). Notice that large $\Gamma$ values match sharper edges. (c): The top two rows show the latent pattern $I$ and the irradiance-based blurred result $B$. The bottom row shows the measured $\Gamma$ which varies across edge strength.

Since $\phi$ has boundary $\phi(0)=0$ and $\phi(1)=1[12]$, we have $\phi(I)=I$. Therefore,

$$
B=\psi(\phi(I) \otimes K)=\psi(I \otimes K)=\psi(\widehat{B}),
$$

and then, $\Gamma=\psi(\widehat{B})-\widehat{B}$. In this toy example, $\Gamma(x)$ simply measures the magnitude how $\psi(x)$ deviates from the linear function $y(x)=x$. In other words, $\Gamma(x) \rightarrow 0$, iff $\psi(x) \rightarrow x$. However, practical CRFs $\psi$ are highly nonlinear [12].

To validate Claim 3 , we synthesize the blurred edges and measure their $\Gamma$ shown in Figure 1(c). The top row shows a sharp pattern corresponding to $I$. Notice that the foreground blocks gradually become brighter from left to right and the rightmost edge has the largest scale of the step-edge. The second row shows its corresponding horizontally motion blurred result $B$. $\phi$ is simulated by Gamma 2.2. The motion length is 20 pixels. The bottom row presents $\Gamma$ and clearly shows that the blurred edge regions $\Gamma$ have significant deviations that cannot be ignored. We also notice that a sharper edge leads to a larger $\Gamma$. Figure 1(b) shows the horizontal profile of the $\Gamma$ used in Figure 1(c). We experiment on four different kernel sizes and show the corresponding $\Gamma \mathrm{s}$. The results illustrate that the magnitude of $\Gamma$ is closely related with the contrast of the edge whereas the kernel size only affects the shape of $\Gamma$ curve.

Finally, we analyze the more general case and provide upper and lower bound to $\Gamma$ :

Theorem 1. Let $I_{\min }$ and $I_{\max }$ be the local lowest and highest pixel intensities in a local neighborhood covered by kernel $K$ in image I. If $\phi$ is convex, then the Blur Inconsistency is bounded by $0 \leq \Gamma \leq I_{\max }-I_{\min }$.

Proof. Consider that $\phi(I) \otimes K$ can be viewed as a convex combination of pixels from $\phi(I)$. If $\phi$ is convex, we can use Jensen's inequality and get

$$
\phi(I) \otimes K \geq \phi(I \otimes K) .
$$

Further, since the CRF $\psi$ is a monotonically increasing [1,13], we have:

$$
B=\psi(\phi(I) \otimes K) \geq \psi(\phi(I \otimes K))=\widehat{B},
$$




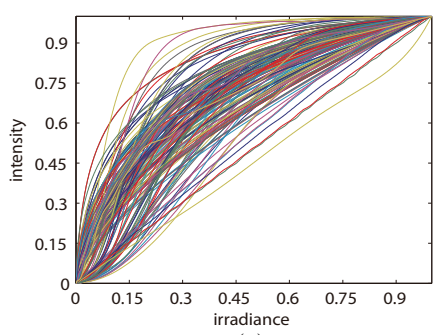

(a)

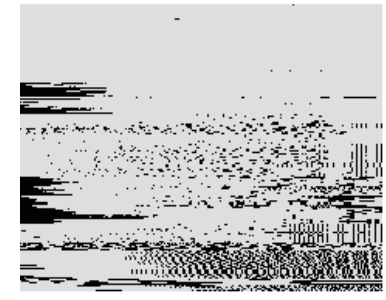

(b)

Fig. 2. The left panel shows 188 CRF curves of real cameras from DoRF [12]. Nearly all curves appear concave. For a clearer illustration, the second-order derivatives at sample points on the curves are plotted on the right. Each row corresponds to a CRF curve where which negative derivatives are drawn in gray and positive in black.

i.e., $\Gamma \geq 0$.

Next, we derive the upper bound of $\Gamma$. Since $I \otimes K \leq I_{\max }$ and $\phi$ is the inversion of $\psi$, it must also be monotonically increasing. Therefore $\phi(I) \otimes K \leq$ $\phi\left(I_{\max }\right)$ and we have,

$$
B=\psi(\phi(I) \otimes K) \leq \psi\left(\phi\left(I_{\max }\right)\right)=I_{\max } .
$$

Likewise, we can also derive taht $\widehat{B}=I \otimes K \geq I_{\min }$. Combining it with Eqn. (14) and Eqn. (15), we have: $I_{\min } \leq \widehat{B} \leq B \leq I_{\max }$. Therefore,

$$
0 \leq \Gamma \leq I_{\max }-\widehat{B} \leq I_{\max }-I_{\min } .
$$

Theorem 1 explains the phenomenon in Figure 1 (b) and (c): when the intensity contrast (gradient) of an edge is high, the upper-bound of $\Gamma$ will be large. In contrast, in low-frequency regions, the upper bound $I_{\max }-I_{\min }$ is lower and so is $\Gamma$, i.e., $B$ can be well approximated by $\widehat{B}$.

It is important to note that Theorem 1 assumes a convex inverse CRF $\phi$. This property has been observed in many previous results. For example, the widely used Gamma curves $\phi(x)=x^{\gamma}, \gamma>1$, are convex. To better illustrate the convexity of $\phi$ or equally the concavity of $\psi$, we plot in Figure 2188 real camera CRF curves $(\psi)$ collected in [12]. We represent all curves by a matrix with 188 rows and compute the discrete second-order derivatives and show the results in the right panel of Figure 2. The negative second-order derivatives are illustrated in gray pixels and the positive ones in black pixels. Our experiment shows that the majority (84.4\%) of sample points are negative and therefore the inverse $\mathrm{CRF} \phi$ is largely convex.

\subsection{Deconvolution Artifacts}

Our theory reveals that if a camera has a non-linear CRF, the conventional intensity-based blur model is largely valid on uniform and smooth regions but fails near high frequency regions such as near occlusion or texture edges. Next, 


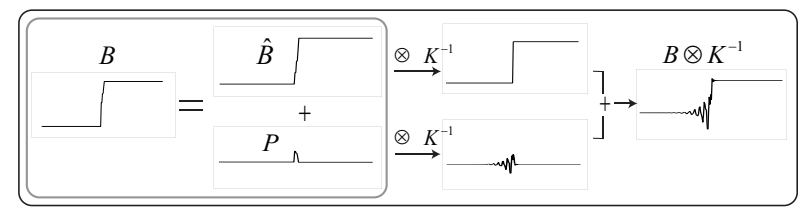

Fig. 3. The ringing artifacts when deblurring a blurred step-edge function. The kernel is invertible and therefore the artifacts are caused by $\Gamma$.

we analyze the deblurring artifacts when applying brute-force intensity-based deblurring method.

In the first example, we analyze the 1D step-edge signal (11) for its simplicity. By 12 , we have $B=\psi(\widehat{B})$. Assume $\psi$ is a Gamma function $\psi(x)=x^{\gamma}, \gamma<1$, we can then expand $B$ with Taylor expansion:

$$
B=\hat{B}^{\gamma}=\hat{B}+P
$$

where $P=\sum_{t=1}^{\infty} \frac{(\gamma-1)^{t}}{t !} \hat{B}(\ln \hat{B})^{t}$. An important property of function $x(\ln x)^{t}$ is that it approaches zero for $x \rightarrow 0^{+}$or $x \rightarrow 1$. Therefore, $P$ only has non-zero values in the blurred edge regions.

Given an invertible filter which has non-zero points in the frequency, we can represent the deconvolution of $B$ with $K$ by $B \otimes K^{-1}$, and thus

$$
B \otimes K^{-1}=\hat{B} \otimes K^{-1}+P \otimes K^{-1},
$$

where $\hat{B} \otimes K^{-1}=I$ since $K$ is invertible. $P \otimes K^{-1}$ can be viewed as the deconvolution artifacts introduced by the Blur Inconsistency $\Gamma$. Figure 3 illustrates the decomposition of (18) in details.

The ringing artifacts in the step-edge function discussed above can also be observed in the frequency domain. Let $I^{*}=B \otimes K^{-1}$. We assume that the fourier coefficient of $K$ at a specific frequency $\omega_{n}$ is $a_{n}$. Therefore, the corresponding coefficient of $K^{-1}$ at frequency $\omega_{n}$ is $1 / a_{n}$. Since $\widetilde{I}$ is a Step Function, its coefficient at $\omega_{n}$ is $\alpha / n$ for $n \neq 0$, where $\alpha$ is a constant value for all $n$. We can further verify that the coefficient of $\widetilde{I} \otimes K$ at frequency $\omega_{n}$ is $\alpha / n \cdot a_{n}$.

If $\psi$ is a linear function, the coefficient of $I^{*}$ at frequency $\omega_{n}$ will be $\beta$. $\alpha / n \cdot a_{n} \cdot 1 / a_{n}=\alpha \beta / n$ where $\beta$ is a constant scaling factor introduced by $\psi$. Therefore, the spectrum of $I^{*}$ will be a scaled version of $\widetilde{I}$, i.e., $I^{*}$ will still be a step function and there will be no ringing artifacts.

In contrast, if $\psi$ is monotonically increasing and concave, Farid [25] proved by using Taylor's series that the coefficients at frequency $\omega_{n}$ for $I^{*}$ will be scaled non-linearly and non-uniformly, i.e., it will no longer be a scaled version of $\alpha / n \cdot a_{n}$ and convolving it with $K^{-1}$ will not cancel out $a_{n}$. As a result, $I^{*}$ will no longer be a step function (edge) but a signal corrupted by non-uniformly scaled high frequencies. Visually it will exhibit strong ringing artifacts.

Finally, we analyze and illustrate the visual artifacts caused by a non-linear CRF in image deblurring. In Figure 4, we synthesize a motion-blurred image 

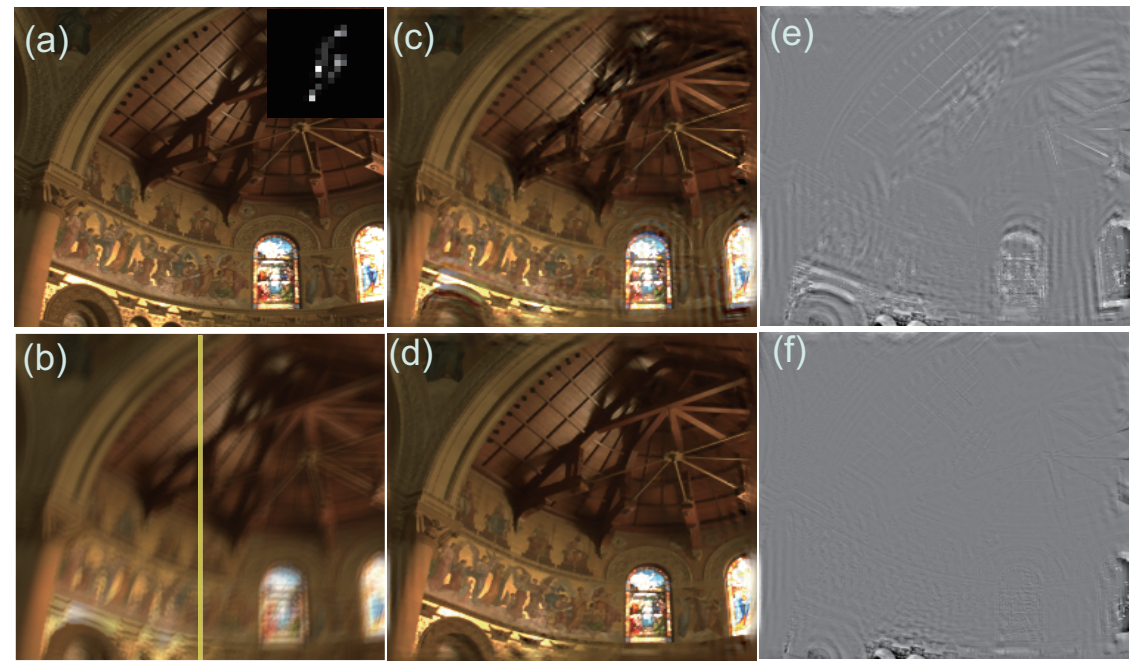

Fig. 4. TV-based deblurring results on the irradiance-based blur image $B$ and on the intensity-based blur image $\widehat{B}$. (a) shows the latent intensity image $I$. (b) shows $B$ (left) and $\widehat{B}$ (right). (c) and (d) show the deblurred results using TV on $B$ and $\widehat{B}$ with the same blur kernel. (e) and (f) show the corresponding error maps.

using an invertible blur kernel under a Gamma 2.2 CRF. Figure 4(a) shows the latent image $I$ and the kernel $K$. (b) shows the irradiance-based blur image $B$ and the intensity-based blur image $\widehat{B}$. We apply non-blind deconvolution using Total Variation regularization [26] to recover the latent images from $B$ and $\widehat{B}$ respectively. The results are shown in Figure 4 (c) and (d). Figure 4 (e) and (f) show the error map to the ground truth.

As shown in Figure 4, the TV-based technique produces high quality result given $\widehat{B}$. However, applying the same deconvolutoin technique on $B$ using the same blur kernel produces ringing artifacts surrounding image edges. Such result is consistent with our Theorem 1. Note that the edges with large contrast $I_{\max }-I_{\min }$ will have large Blur Inconsistency $\Gamma$, and exhibit noticeable ringings artifacts.

Theorem 1 reveals the importance of image regularization in intensity-based deblurring. When images have moderate edge contrasts, our experiments show that Total Variation regularized nonblind deconvolution can produce reasonable results. However, for images with large edge contrasts, the Blur Inconsistency will be large and suppressing the ringing artifacts can be difficult. The deconvolution models used $[5,7]$ further help to suppress ringing by assuming heavy-tailed distribution, local gradient constraint, noise distribution, etc.

\section{Dual-Image CRF Estimation}

Based on our analysis, we present a new computational photography technique to estimate the CRF using a pair of images. Our work is inspired by the recent dual- 
image processing techniques where a pair of images captured towards the same scene but under different aperture/shutter settings are used [4]. In a similar vein, we use a blurry/sharp image pair: the first image is captured with a slow shutter and introduces motion blurs whereas the second is captured with fast shutter but high ISO. The main difference is that [4] uses known CRF for deblurring whereas our goal is to estimate CRF from the image pair.

Recall that our analysis shows that the blurred edge pixels reveal most information about the CRF. We therefore focus on using these pixels. We first align the two images and estimate the blur kernel. Next, we approximate the CRF by fitting a non-linear function to match the edge pixels on the blur-free image to the corresponding ones on the blurred image. Compared with the recent singleimage CRF estimation technique [9] that relies on 1D linear motion blurs, our solution aims to provide a more flexible setup, i.e., using a hand-held camera and it can handle complex 2D motions.

We apply the kernel sparsity based method [27] to simultaneously register the blurry/sharp image pair and estimate the kernel. In our setup, we capture the images with nearly identical exposure settings of the camera pair (i.e., $0.8 \leq$ $r \leq 1$ ) by properly adjusting the shutter and the ISO. This allows us to robustly register the pair. Finally, to recover the CRF, we model the inverse CRF $\phi(x)$ using the Generalized Gamma Curve Model (GGCM) [28]:

$$
\phi(x)=x^{1 / P(x, \alpha)}, P(x, \alpha)=\sum_{i=0}^{n} \alpha_{i} x^{i} .
$$

In our experiment, we find that $n=4$ is usually accurate enough to reproduce the CRF.

To find the optimal $\phi$, the brute-force approach is to minimize the difference between prediction and observation in the irradiance domain:

$$
\|\phi(B)-\phi(I) \otimes K \cdot r\|^{2} .
$$

Apparently, a trivial solution is $\phi(x)=0$. We therefore set out to minimize the difference in the intensity domain as:

$$
\|\psi(\phi(I) \otimes K \cdot r)-B\|^{2} .
$$

Claim 1-3 show that the edge pixels contribute most information to the CRF. Therefore, instead of treating all pixels equally, we assign more weights to pixels near edges that have a high inconsistency measure $\Gamma$. The final weighted energy function is then:

$$
J(\phi)=\|W \cdot(\psi(\phi(I) \otimes K \cdot r)-B)\|^{2},
$$

where the weighting matrix $W$ is determined by

$$
W(i, j)= \begin{cases}\Gamma(i, j) & \text { if } \Gamma(i, j)>\tau_{1} \\ 0 & \text { else. }\end{cases}
$$


If the two images have slightly different exposures, i.e., $r \neq 1$, we will not be able to directly measure $\Gamma$. In this case, we measure the upper-bound of $\Gamma(16)$ to define the weight matrix as:

$$
W(i, j)= \begin{cases}I_{\max }-I_{\min } & \text { if } I_{\max }-I_{\min }>\tau_{2} \\ 0 & \text { else. }\end{cases}
$$

In our experiments, we use $\tau_{1}=0.02, \tau_{2}=0.2$ as the edge threshold. To improve robustness, we further exclude saturated pixels in our optimization. Finally, the computed exposure ratio $r$ from the camera parameters can still contain errors due to camera hardware controls. To reduce error, we calculate initial exposure ratio according to exposure time and ISO value and then iteratively update $\phi$ and $r$ where $r$ is obtained by fitting the observation model Eqn.(4):

$$
r=\frac{\sum_{i, j}(\phi(I(i, j)) \otimes K) \phi(B(i, j))}{\sum_{i, j}(\phi(I(i, j)) \otimes K)^{2}}
$$

Since $\phi$ and its inverse function $\psi$ both appear in the energy function, the direct optimization is difficult. We apply non-linear Nelder-Mead Simplex method [18] to find the optimal solution.

\section{$5 \quad$ Experiments and Results}

We have evaluated our dual-image based CRF estimation technique on both synthesized images and real cameras. For synthetic results, we use the ground truth irradiance image $\widetilde{I}$ and a known blur kernel $K$ to generate a blurred irradiance image $\widetilde{B}$. We then map $\widetilde{I}$ and $\widetilde{B}$ back onto the corresponding intensity images $I$ and $B$ using predefined CRFs. Specifically, we select the first ten camera CRFs listed in [13] and use only the green channel CRF.

To synthesize motion blurs, we use eight canonical PSFs from Levin et al. [19] as the blur kernels. To test robustness, we further add noise to the latent intensity image $I$. The addition of noise is important to faithfully emulating capturing images under a high ISO. We use Gaussian noise with three different variances $\sigma^{2}=0.001,0.005$ and 0.01 (with image intensity scaled to $[0,1]$ ). This produces a total of 240 possible combinations in terms of CRFs, blur kernels, and noise levels.

Next, we apply our CRF estimation algorithm to all 240 cases. The input of the algorithm includes $B$, noisy $I$ and blur kernel $K$. Then we measure the error of the recovered CRFs. Figure 5 shows our results for a specific $\phi$ illustrated as the red curve. The left panel shows our estimated $\phi$ at three noise levels and the right panel shows the average RMSE (Root Mean Square Error) of our estimations over all 240 estimated curves. Our estimations at different noise levels (shown in three different colors) have similar mean RMSE: 0.0155, 0.0137 and 0.0169 respectively. This illustrates that our technique is robust in presence of image noise. 


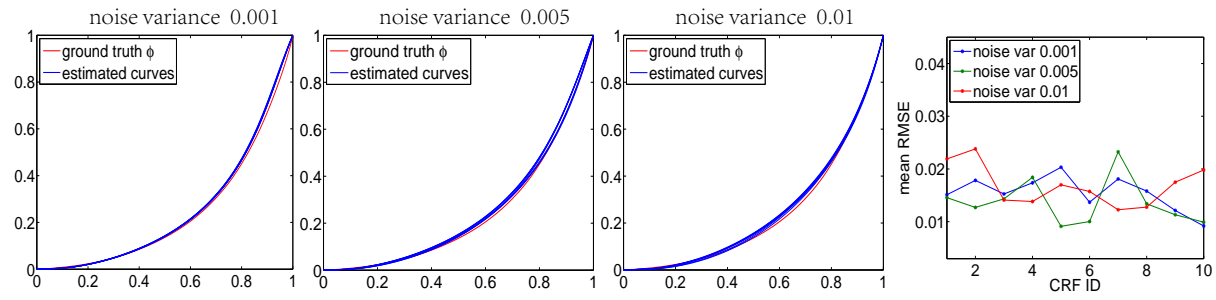

Fig. 5. CRF estimation on synthetic images. The left three panels show the results on a specific CRF with different noise levels. At each noise level, the red curves shows the ground truth $\mathrm{CRF}$ and the blue curves show our estimation results under 8 different blur kernels. We average the RMSE between the estimated ones and the ground truth as the error. The right panel shows the error curve of 10 different CRFs.
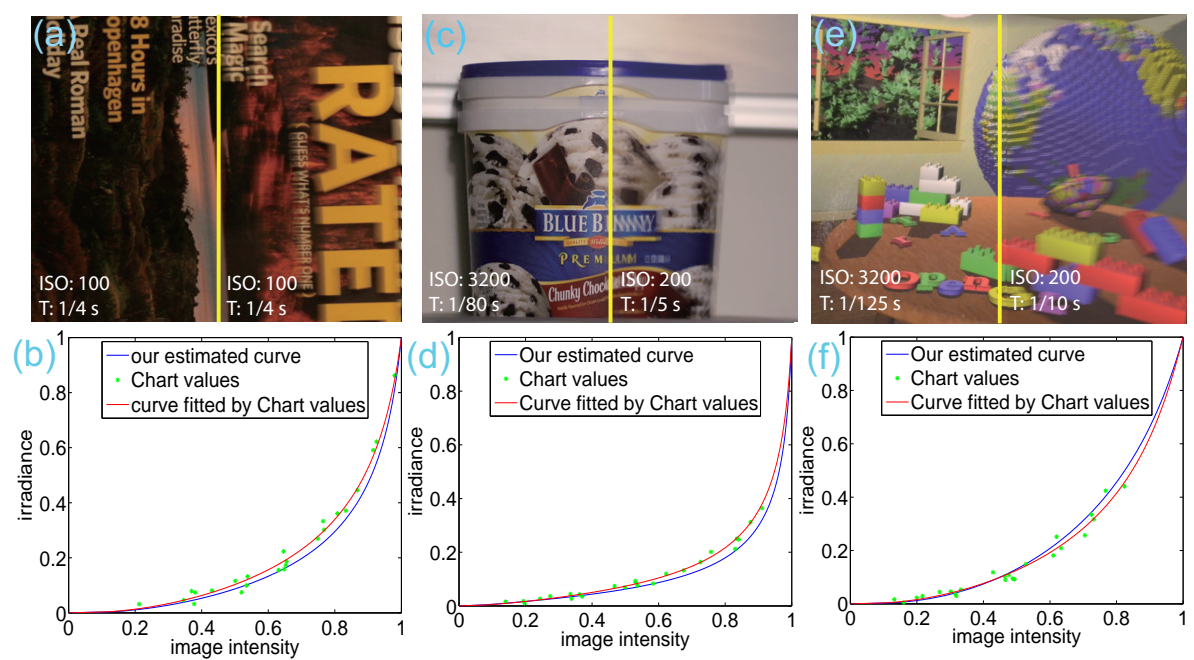

Fig. 6. CRF estimation on real images. The top row shows the sharp/blurred pairs. The bottom row shows our recovered the CRF (in blue) and the ground truth CRF (in red) obtained by acquiring the MacBeth's chart (in green).

We have also validated our approach on three real cameras: Canon 60D, 400D, and Nikon D3100. Figure 6 (a), (c) and (e) show sample captured sharp/blurry image pairs. We also list the ISO and exposure setting for each captured image. The sharp image in (a) is captured with a tripod whereas the rest are captured by holding the camera by hands to introduce motion blurs. We recover the blur kernel using [4] and then apply our algorithm to estimate $\phi$. To obtain the ground truth $\phi$, we further capture an image of the Macbeth color checkerboard and apply the PCA-based method [12] to estimate $\phi$ via curve fitting [28]. Figure $6(\mathrm{~b}, \mathrm{~d}, \mathrm{f})$ plot our recovered CRFs against the ground truth ones. Our technique is able to achieve comparable quality. 


\section{Discussions and Future Work}

We have presented a comprehensive study to analyze the effect of the Camera Response Function (CRF) in motion blurring. We have shown that for non-linear CRFs, the intensity-based and irradiance-based blur models are similar at low frequency regions but are significantly different at high frequency regions such as edges. Our theory shows that directly applying deconvolution on the intensity image leads to strong ringing artifacts that are irrelevant to kernels. Based on our analysis, we have developed a dual-image solution that captures a pair of sharp/blurred images with a hand-held camera to simultaneously recover the CRF and to deblur the image.

Although our solution uses a much simpler setup than traditional multiimage based techniques, our method has a number of limitations. The algorithm relies on accurately registering two images captured under different exposure settings. In our implementation, we directly use sparsity-based methed [27] that was originally proposed to register two relatively low dynamic range images with similar appearances. In our case, the sharp/blurry images would be captured under high dynamic range for recovering the CRF and the two images can appear significantly different due to exposure variations. Consequently, the recent singleimage based solution [10] has a key advantage.

Another important future direction that we will explore is to deblur images without knowing the CRF. Our analysis shows that applying brute-force deconvolution will introduce ringing artifacts even if the kernel is invertible. One possible solution is to first detect potential ringing regions in the deblurred result and then analyze if they are caused by a non-linear CRF. Finally, we will explore possible integrations of our analysis/approach with the single-image approach $[10]$.

Acknowledgments. This project was partially supported by the National Science Foundation (US) under grants IIS-CAREER-0845268 and IIS-RI-1016395; Air Force Office of Science Research under the YIP Award; Natural Science Foundation (China) (Nos:61105001, 61075012) and the Committee of Science and Technology, Shanghai (No. 11530700200). This work was done when Chen was a visiting scholar at the University of Delaware.

\section{References}

1. Debevec, P.E., Malik, J.: Recovering high dynamic range radiance maps from photographs. In: SIGGRAPH. (1997)

2. Chang, Y.C., Reid, J.F.: RGB calibration for color image-analysis in machine vision. IEEE Trans. on IP 5 (1996) 1414-1422

3. Fergus, R., Singh, B., Hertzmann, A., Roweis, S.T., Freeman, W.T.: Removing camera shake from a single photograph. In: SIGGRAPH. (2006)

4. Yuan, L., Sun, J., Quan, L., Shum, H.Y.: Image deblurring with blurred/noisy image pairs. SIGGRAPH (2007) 
5. Shan, Q., Jia, J., Agarwala, A.: High-quality motion deblurring from a single image. SIGGRAPH (2008)

6. Cho, S., Lee, S.: Fast motion deblurring. ACM SIGGRAPH Asia '09 (2009)

7. Chen, X., He, X., Yang, J., Wu, Q.: An effective document image deblurring algorithm. In: CVPR. (2011)

8. Chen, X., Yang, J., Wu, Q.: Image deblur in gradient domain. Optical Engineering 49 (2010) $117003+$

9. Wilburn, B., Xu, H., Matsushita, Y.: Radiometric calibration using temporal irradiance mixtures. CVPR (2008)

10. Kim, S., Tai, Y.W., Kim, S., Brown, M.S., Matsushita, Y.: Nonlinear camera response functions and image deblurring. In: CVPR. (2012)

11. Kim, S.J., Pollefeys, M.: Robust radiometric calibration and vignetting correction. IEEE Trans. PAMI 30 (2008) 562-576

12. Grossberg, M.D., Nayar, S.K.: Modeling the space of camera response functions. IEEE Trans. PAMI 26 (2004) 1272-1282

13. Mitsunaga, T., Nayar, S.K.: Radiometric self calibration. CVPR (1999)

14. Lee, J.Y., Shi, B., Matsushita, Y., Kweon, I.S., Ikeuchi, K.: Radiometric calibration by transform invariant low-rank structure. In: CVPR. (2011)

15. Kim, S., Frahm, J., Pollefeys, M.: Radiometric calibration with illumination change for outdoor scene analysis. In: CVPR. (2008)

16. Lin, S., Gu, J., Yamazaki, S., Shum, H.Y.: Radiometric calibration from a single image. CVPR (2004)

17. Lin, S., Zhang, L.: Determining the radiometric response function from a single grayscale image. CVPR (2005)

18. Matsushita, Y., Lin, S.: Radiometric calibration from noise distributions. CVPR (2007)

19. Levin, A., Weiss, Y., Durand, F., Freeman, W.: Understanding and evaluating blind deconvolution algorithms. CVPR (2009)

20. Tai, Y.W., Du, H., Brown, M.S., Lin, S.: Correction of spatially varying image and video motion blur using a hybrid camera. IEEE Trans. PAMI 32 (2010) 1012-1028

21. Joshi, N., Szeliski, R., Kriegman, D.J.: Psf estimation using sharp edge prediction. CVPR (2008)

22. Jia, J.: Single image motion deblurring using transparency. In: CVPR. (2007)

23. Krishnan, D., Tay, T., Fergus, R.: Blind deconvolution using a normalized sparsity measure. In: CVPR. (2011)

24. Weiss, Y., Freeman, W.T.: What makes a good model of natural images? CVPR (2007)

25. Farid, H.: Blind inverse gamma correction. IEEE Transactions on Image Processing 10 (2001) 1428-1433

26. Wang, Y., Yang, J., Yin, W., Zhang, Y.: A new alternating minimization algorithm for total variation image reconstruction. SIAM J. Img. Sci. 1 (2008) 248-272

27. Yuan, L., Sun, J., Quan, L., Shum, H.Y.: Blurred/non-blurred image alignment using sparseness prior. ICCV (2007)

28. Ng, T.T., Chang, S.F., Tsui, M.P.: Using geometry invariants for camera response function estimation. In: CVPR. (2007) 